Please do not remove this page

RMIT

UNIVERSITY

\title{
Transmission of compressed multimedia data over wireless channels using space-time OFDM with adaptive beamforming
}

Athanasiadis, Tasso; Lin, Kevin; Hussain, Zahir

https://researchrepository.rmit.edu.au/esploro/outputs/9921858796201341/filesAndLinks?institution=61RMIT_INST\&index=null

Athanasiadis, T., Lin, K., \& Hussain, Z. (2005). Transmission of compressed multimedia data over wireless channels using space-time OFDM with adaptive beamforming. Proceedings of the TENCON 2005 IEEE Region 10 Conference. https://doi.org/10.1109/TENCON.2005.301344

Published Version: https://doi.org/10.1109/TENCON.2005.301344

Repository homepage: https://researchrepository.rmit.edu.au

(c) 2005 IEEE. Personal use of this material is permitted. However, permission to reprint/republish this material for advertising or promotional purposes or for creating new collective works for resale or redistribution to servers or lists, or to reuse any copyrighted component of this work in other works must be obtained from the IEEE.

Downloaded On 2023/04/26 13:41:54 +1000 


\title{
Transmission of Compressed Multimedia Data over Wireless Channels using Space-time OFDM with Adaptive Beamforming
}

\author{
Tasso Athanasiadis, Kevin H. Lin, IEEE, Student Member, and Zahir M. Hussain, IEEE, Senior Member \\ School of Electrical and Computer Engineering, RMIT University, Melbourne, Victoria 3000, Australia. \\ E-mails: s8902991@ student.rmit.edu.au; s9510490@student.rmit.edu.au; zmhussain@ieee.org
}

\begin{abstract}
The transmission of multimedia data over wireless channels poses significant constraints on the communication system bandwidth, energy, and latency. To overcome these bottlenecks to wireless multimedia communication, various channel coding and transmit diversity schemes have been proposed. In previous work, we have shown that space-time block-coding (STBC) with adaptive beamforming (STBC-OFDM-AB) is an effective technique for improving the error-rate performance and channel capacity of wireless multimedia systems utilizing OFDM. In this paper, we introduce a transmission system for multimedia communication employing STBC-OFDM with adaptive beamforming incorporating a perceptually-based image compression coder - which consists of a 2-D discrete wavelet transform (DWT), an adaptive quantizer (with thresholding) and variable-length entropy encoding. Initial simulation results based on the transmission of compressed images, showed that the performance improvement introduced by STBC-OFDM-AB can be readily observed, and compared to other transmission methods is better suited to wireless multimedia communication.
\end{abstract}

\section{INTRODUCTION}

Over the past decade, wireless multimedia communication has attracted considerable interest by both the research community and industry to address the increased demand for multimedia services over wireless networks such as $3 \mathrm{G}$ mobile communication systems. Recent trends in next-generation (NG) wireless/mobile networks [1] suggest a significant shift towards personalized mobile communications - ubiquitous computing - and a stronger need for wireless multimediaequipped devices capable of high data rate transmission. This is mainly due to a significant increase in the amount of wireless multimedia content transmitted over traditional telephonyoriented systems, which have predominantly supported the transmission of voice data.

In a wireless environment, the time-varying nature of channel conditions and propagation characteristics, such as multipath fading, inherently subject wireless signals to intersymbol interference (ISI) leading to high bit error rates (BER) [2]. Moreover, the transmission of wireless data over bandlimited multipath fading channels cause a degradation in both communication channel performance, system latency, and the acceptable or subjective quality of data received on wireless terminals/devices.

It is well known, that the communication bandwidth available to wireless/mobile systems, and the processing capabil- ities of the communication system/devices are often severely limited. Mobile radio channels must, therefore, transmit user information in a highly compressed form, while making efficient use of available frequency spectrum and communication power. As a result, the transmission of multimedia data over wireless communication channels introduces significant bottlenecks. This is mainly due to the fact that representing multimedia data such as digital audio/images/video requires a large amount of information, leading to high bandwidth, computation energy, and communication energy requirements.

To deal with the transmission of multimedia data over wireless communication networks several key compression technologies have been developed - JPEG2000, MPEG, H.263/L [3]. The processing of high-quality digital video, images, and audio signals using quite sophisticated and advanced compression algorithms (codecs) are at the heart of many modern communication products, from personal digital assistants (PDAs) to video-capable cell phones. However, to support multimedia transmission through noisy wireless/mobile radio environments various 'adaptive' techniques (by dynamically controlling communication parameters to suit variations in channel conditions, computational requirements, and available processing resources) have emerged. For example, in [4], the authors adapt source coding parameters of the JPEG image compression algorithm on energy consumption, bandwidth, and latency. A methodology to select the optimal image compression parameters to minimize communication energy consumption given the current channel conditions and constraints is also proposed. In [5], the authors use a joint source/channel coding scheme based on turbo-coded OFDM to transmit compressed images over a band limited frequency selective fading channel.

Moreover, in recent years, transmit diversity techniques such as space-time block coding (STBC) has attracted great attention for its capability to enhance the error performance and the capacity of wireless systems [6][7]. Further improvements to system performance by combining STBC with adaptive beamforming, in a spatially correlated channel environment, can be achieved as shown in [8]. This technique has been extended for use in broadband OFDM systems [9]. In [10], we have shown that the use of space-time coded OFDM system with an adaptive eigenbeamforming scheme (STBC-OFDM- 


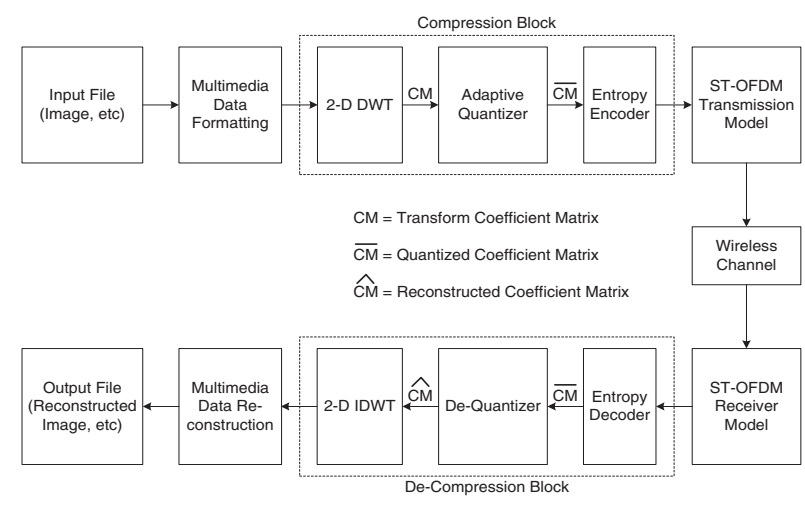

Fig. 1. Block diagram of the transmission system model

$\mathrm{AB}$ ) is well suited to the application of multimedia data, and that a significant BER perfomance gain readily facilitates the transmission of high-quality/high data-rate multimedia signals.

In this paper, we present a transmission system for the application of compressed images over wireless channels using a combined source coding and STBC-OFDM-AB scheme. This method can be extended to study the transmission of other sources of multimedia information such as digital audio and video data. Subsequent sections of this paper have been organized as follows: Section 2, provides an overview of the system model proposed and describes the transmission elements; Simulation results are given in Section 3, along with a discussion of the overall achievements obtained from this work; Section 4 discusses future directions and concludes the paper.

Notation used in this paper: $(.)^{T}$, and $(.)^{H}$ are vector and Hermitian transpositions, respectively. $\|\mathcal{A}\|_{F}$ and $\sqrt{\mathcal{A}}$ denote the Frobenius norm and Hermitian square root of matrix $\mathcal{A}$. Finally, capital bold letters represent matrices, and small bold letters are column vectors.

\section{System ModeL}

The elements of the transmission system model proposed in this paper, consist mainly of 3 blocks: multimedia data formatting, data compression, and an adaptive ST-OFDM model. Note, that a channel encoder such as turbo coding is not employed, as an initial baseline performance of the system is required. Channel encoding schemes provide forward error correction and only add redundancy to the entropy coded data to achieve a BER close to the shanon limit. A block diagram of the system model is shown in Figure 1. This is based on the STBC-OFDM-AB simulation scheme previously outlined in [10]. A description of the main blocks is provided in the following sections.

\section{A. Modeling of Multimedia Data}

To enable the simulation of multimedia (image) data over the adaptive space-time OFDM transmission scheme, multimedia signals from various data sources (such as, compressed audio/ image/ video files), which may be stored on a PC, are converted to a suitable format which can be further processed by the compression block (transform/entropy coder).

The principal idea behind the multimedia data model developed is to consider the source file (digitized version of the original multimedia signal with n-bit resolution) as a vector array containing integer quantization levels. For digital audio, these correspond to sampled amplitude values, and for digital image and video coding they correspond directly to pixel values. In the case of monochrome digital still images (with 8-bit/pixel resolution), the source data is converted into a matrix array $(M \times N)$ containing 8-bit integer values which correspond to intensity information of the image.

The output - after transform/subband coding, adaptive quantization, and entropy coding - of the compression block provides a vector array of integers representing the bitstream required for conversion to a vector array of baseband modulated data symbols $\{s(n)\}_{n=0}^{N_{s}-1}$, as specified by the spacetime OFDM model. In the receiver path, the reverse operation is performed on the modified data symbols $\{\hat{s}(n)\}$ produced by the space-time OFDM transmission scheme. Whereby, the data symbols are first converted back to a bitstream and subsequently to an integer array, which can be reconstructed (via inverse operations by the de-compressor) back to the original multimedia data format applied to the system.

\section{B. Data Compression}

Referring to Figure 1 signal compression of image data consists of the following steps:

1) Wavelet decomposition using 2D-DWT.

2) Adaptive quantization, with threshold.

3) Entropy coding (Huffman coder).

The use of the discrete wavelet transform (DWT) in the compression of multimedia signals, such as image/video compression applications, has been well documented [11] [12]. For example, wavelet transform coding has been accepted as the core processing tool in the JPEG2000 image compression standard, as it has a number of advantages over other compression techniques (DCT subband coding, vector quantization, fractal coding, etc). Some of the main benefits of wavelet transform coding include, the high decorrelation and energy compaction capability, reduction in image distortion due to blocking artifacts and mosquito noise, and the adaptive spatial-frequency resolution offered which is well suited to the properties of the human visual system (HVS) - psychovisual redundancy (HVS properties which can be exploited to achieve superior compression). In general, image compression applications based on wavelet transform coding provide better image quality over other subband coding techniques such as block DCT, particularly, for large compression ratios [13].

In the case of transmitting digital images, a multiresolution 2D-DWT decomposition based on dyadic scales and positions (dilations and translations) of the wavelet basis function (mother wavelet) is obtained using two subband filters. After each level of decomposition four bands of data are produced, one corresponding to the low-pass band and three others corresponding to horizontal, vertical, and diagonal high-pass 
bands - with all subbands providing spatial and frequency information simultaneously. This subband information can be iteratively decomposed resulting in multiple 'transform levels'. In theory this process can be continued indefinitely, however, in practice a suitable number of levels is chosen based on a suitable entropy-based criterion.

In wavelet analysis, we represent the low-resolution subband by approximation coefficients $\left(\mathbf{c A}_{\mathbf{n}}\right)$ and high-pass subband spatial-frequency information with horizontal, vertical, and diagonal detail coefficients $\left(\mathbf{c H}_{\mathbf{n}}, \mathbf{c V}_{\mathbf{n}}, \mathbf{c D}_{\mathbf{n}}\right)$ respectively. For an n-level decomposition, the coefficient matrix $\mathbf{C M}$ consists of the following coefficients, $\mathbf{C M}=$ $\left[\mathbf{c A}_{\mathbf{n}}, \mathbf{c H}_{\mathbf{n}}, \mathbf{c V}_{\mathrm{n}}, \mathbf{c D}_{\mathrm{n}}, \cdots, \mathbf{c H}_{1}, \mathbf{c V}_{1}, \mathbf{c D}_{1}\right]$.

During transmission, the transform coefficients (DWTC) are adaptively mapped to a finite set of values using a uniform scalar quantizer. Spatial redundancy of the detail coefficients can be exploited, as many of the coefficients will be either small in value or contain a high number of elements close to or equal to zero. A threshold can be adaptively set to remove coefficients below a preset value. Since the statistics of the detail DWTC can vary, a different threshold can be applied independently to each band/level or a 'global' threshold parameter for all subbands can be applied. For lossless compression, the DWTC are quantized with their actual values. In contrast, however, to achieve high compression ratios a lossy compression technique which uses an aggressive quantization scheme is required. After the data has been adaptively quantized, additional lossless compression is achieved by further removing spatial and statistical redundancy of the DWTC using a run-length and entropy encoding respectively. In this paper, we use an entropy coder based on an improved Huffman coding scheme using recursive splitting of symbol sequences [14].

\section{The Adaptive Space-Time OFDM Model}

The general structure of the ST-OFDM transmission model with adaptive eigenbeamforming in a MIMO channel configuration employing $N_{t}$ and $N_{r}$ antennas at the transmitter and receiver, is shown in Figure 2. The OFDM system we consider in this paper utilizes $N_{c}$ frequency tones. The channel is assumed to be frequency-selective having quasi-static fading. At the transmitter, a sequence of $N_{s}$ multimedia baseband modulated data symbols $\left\{s_{k}(n)\right\}_{n=0}^{N_{s}-1}$ is first orthogonal space-time block (OSTB) encoded into codeword matrices $\left\{\mathbf{C}_{k} \mid k=1, \cdots, N_{c}\right\} \in \mathcal{C}^{p \times N_{t}}$, which spans across $N_{t}$ spatial dimensions and $p$ adjacent OFDM symbol intervals. $\mathbf{C}_{k}$ is the OSTB encoded output matrix to be transmitted on the $k^{\text {th }}$ subcarrier and $\mathcal{C}$ denotes all possible codeword matrices that can be constructed. Before the stage of inverse Fourier transformation (IFFT) OSTB codeword $\mathbf{C}_{k}$ is linearly transformed by the adaptive beamforming weights as shown in Fig. 2.

For the $N_{t}$ BS antennas, we assume a uniform linear array (ULA) configuration is used with a element spacing of $d$ meters apart. In general, the spacing between the elements of ULA at the transmitter is not large due to the condition at the antenna site to have zero cross-correlation factor, a typical scenario in cellular network. Therefore, spatial correlation between antenna elements would be effective. This correlation is defined in [15] based on the geometric scenario characterized by AoA. To model this spatial correlation, we let the $\ell^{\text {th }}$ path signal impinging on an ULA have an angleof-arrival (AoA) of $\phi_{\ell}$ during reverse link signalling. Then the array propagation vector can be expressed as $\underline{a}\left(\phi_{\ell}\right):=$ $\left[1, e^{j \beta}, e^{j 2 \beta}, \cdots, e^{j\left(N_{t}-1\right) \beta}\right]^{T}$ where $\beta=\left(2 \pi \cdot d \cdot \sin \left(\phi_{\ell}\right)\right) / \lambda$ and $\lambda$ being the carrier frequency wavelength. According to [15], the spatial covariance matrix that specifies the spatial correlation between antenna elements is given by:

$$
\mathbf{R}_{t}=\frac{1}{L} \sum_{\ell=0}^{L-1} \mathbf{a}\left(\phi_{\ell}\right) \mathbf{a}^{H}\left(\phi_{\ell}\right)
$$

where $L$ denotes the number of dominant resolvable paths. In this paper, we assume that spatial channel correlation at the receiver side is zero, hence, only the transmitter side will be considered. This means that $\mathbf{R}_{r}=\mathbf{I}_{N_{r}}$, where $\mathbf{I}_{M}$ is an identity matrix having a size of $M \times M$.

Denote $\mathbf{h}_{i, j}=\left[h_{i, j}(0), \cdots, h_{i, j}(L-1)\right]$ to be the $L$-tap channel impulse response vector between $(i, j)^{\text {th }}$ transmitreceive antenna pair. The elements of this vector are modelled according to the geometrical-based hyperbolically distributed scatterers (GBHDS) channel model in [16] with Jakes power spectral density. The spatially correlated frequency response matrix for the $k^{\text {th }}$ subcarrier frequency can then be expressed as $\mathbf{H}_{k}=\sqrt{\mathbf{R}_{t} \overline{\mathbf{H}}_{k}}$, where the $(i, j)^{\text {th }}$ element of $\mathbf{H}_{k}$ is given by $\mathbf{H}_{k}(i, j)=\mathbf{h}_{i, j} \mathbf{f}_{k}$ and $\mathbf{f}_{k}=$ $\left[1, e^{-j 2 \pi(k-1) / N_{c}}, \cdots, e^{-j 2 \pi(k-1) \tau_{L-1} / N_{c}}\right]^{T} \in \mathbb{C}^{L \times 1}$ is the corresponding discrete Fourier transform coefficients. $\overline{\mathbf{H}}_{k} \in$ $\mathbb{C}^{N_{t} \times N_{r}}$ is the uncorrelated channel fading gain for the $k^{\text {th }}$ subcarrier.

Maximizing the signal power along the dominant multipaths of the channel, eigen-decomposition of the spatial correlation matrix should be performed. The eigen-decomposition has the following form:

$$
\mathbf{R}_{t}=\mathbf{V D V}^{H}
$$

where $\mathbf{D}:=\operatorname{diag}\left(\lambda_{d}, \lambda_{2}, \cdots, \lambda_{N_{t}}\right)$ is a diagonal matrix with ordered eigenvalues and $\mathbf{V}:=\left[\boldsymbol{v}_{1}, \cdots, \boldsymbol{v}_{N_{t}},\right]$ is a unitary matrix composed of the corresponding eigenvectors. To facilitate signal transmission in the eigen-modes of the correlation matrix, eigen weight mapping is performed across the space dimension of the OSTB codewords $\left\{\mathbf{C}_{k}\right\}_{k=1}^{N_{c}}$ prior to transmission. Mathematically, it can be expressed as $\mathbf{W}^{H} \mathbf{C}_{k}$, where $\mathbf{W}=\left[\mathbf{w}_{1}, \cdots, \mathbf{w}_{N_{t}}\right]$ is the eigen weight mapping matrix and $\mathbf{w}_{i}=\boldsymbol{v}_{i}$. Hence, signal transmission on different eigenvectors of $\mathbf{R}_{t}$ amounts for transmitting $N_{t}$ orthonormal beams in the direction of the dominant multipaths seen by the BS. Then the discrete time baseband equivalent expression of the combined received signal has the form:

$$
\mathbf{Y}_{k}=\mathbf{C}_{k} \mathbf{W}^{H} \mathbf{H}_{k}+\mathbf{E}_{k},
$$

where $\mathbf{E}_{k}$ is a additive Gaussian noise matrix at the receiver with each element having zero mean and $\sigma_{k}^{2}$ variance. 


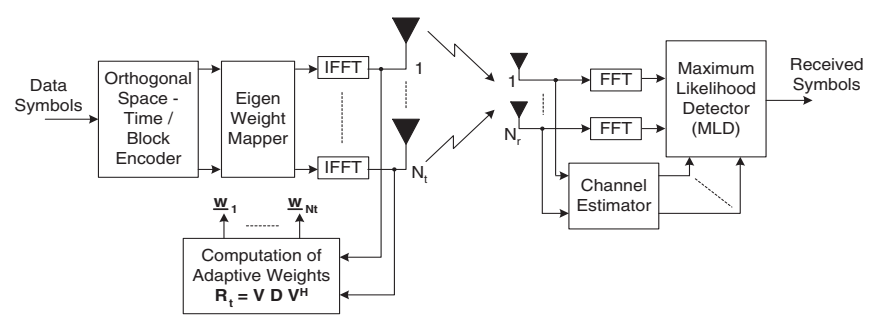

Fig. 2. General structure of the ST-OFDM transmission model with adaptive eigenbeamforming.

At the receiver, channel estimation is performed by evaluating pilot tones embedded in the transmitted signal, then it is fed into the maximum likelihood detector (MLD) for OSTB decoding of data symbols. Assume that the receiver has an ideal knowledge of the channel state information. To decode the received signal matrix, the MLD will evaluate the decision matrix and decide in favor of the codeword as follows:

$$
\widehat{\mathbf{C}}_{k}=\arg \min _{\mathbf{C}_{k} \in \mathcal{C}} \sum_{k=1}^{N_{c}}\left\|\mathbf{Y}_{k}-\mathbf{C}_{k} \mathbf{W}^{H} \mathbf{H}_{k}\right\|_{F}^{2} .
$$

The multimedia information data can then be recovered through baseband demodulation of the estimated OSTB codeword $\widehat{\mathbf{C}}_{k}$. The adaptive ST-OFDM system performance and thus the resulting quality of the multimedia data depends on the estimation accuracy of the decision matrix (4).

\section{Simulation Results}

To evaluate the performance of the transmission system for image applications, a standard version of the $512 \times 512$ size gray scale (monochrome) Lena image with 8 bits/pixel resolution, is used as the multimedia source data/file input to the simulation framework. Due to practical computational limitations, however, the Lena image is resized to $128 \times 128$ using 'bilinear' interpolation. This introduces only slight imperfections in the image (visual quality). The re-sized Lena image used for source coding is shown in Figure 3. This was transformed using biorthogonal wavelets, which are needed for image reconstruction to maintain a linear phase, and a level 4 wavelet decompostion was performed.

The space-time OFDM model is configured for QPSK baseband modulation, using the ST encoding matrix $\mathcal{G} 4$ in [7], and a GBHDS correlated macrocell channel model developed in [16]. Also, the source coder (compression block) was configured to provide a compression ratio (CR) of 20.21 using a DWTC threshold of 10, and the Huffman coder in [14]. In order to determine the effectiveness of the adaptive space-time OFDM system over other methods, the framework was simulated and compared for two cases: OFDM with no space-time coding or adapative beamforming and a space-time coded OFDM system with adaptive beamforming. Objective performance metrics commonly used in image processing literature, such as the MSE and PSNR, was recorded for both cases using an initial system SNR of $20 \mathrm{~dB}$, and subsequently repeated for a SNR of $10 \mathrm{~dB}$.

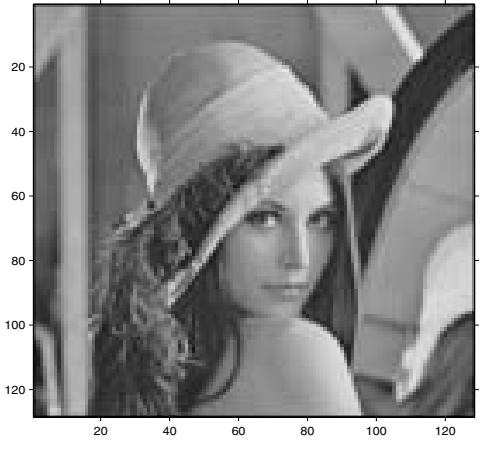

Fig. 3. Original Lena source image (8-bit gray scale) resized to $128 \times 128$.

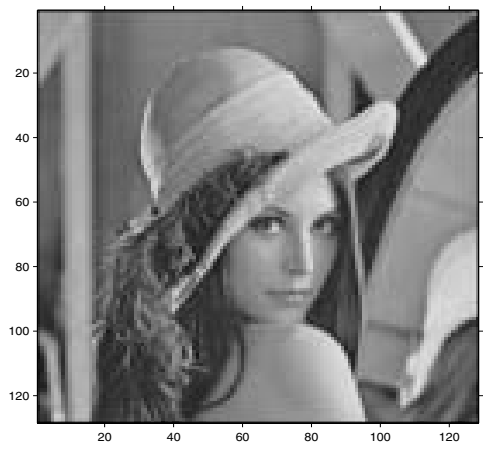

Fig. 4. Reconstructed Lena image from the OFDM system with space-time coding and adaptive beamforming: $\mathrm{SNR}=20 \mathrm{~dB}$

For the case where an OFDM system model with no space-time coding or adaptive beamforming is utilised, the MSE/PSNR degrades from a value of $\mathrm{MSE}=4.62 / \mathrm{PSNR}=41.48 \mathrm{~dB} \quad$ (at $\mathrm{SNR}=20 \mathrm{~dB})$ to a $\mathrm{MSE}=1376.27 / \mathrm{PSNR}=16.74 \mathrm{~dB} \quad$ (at $\mathrm{SNR}=10 \mathrm{~dB}$ ). When the transmission system is configured for space-time OFDM system with adaptive beamforming, the MSE/PSNR remains the same at MSE $=0.22 / \mathrm{PSNR}=54.77 \mathrm{~dB}$ for both values of SNR.

These results are confirmed by inspecting the subjective quality of the images obtained, as shown in the accompanying Figures 4, 5, 6, and 7. Visual inspection and comparison of the received Lena images at a SNR of $10 \mathrm{~dB}$, shows severe image distortion is introduced by OFDM systems with no space-time coding or adaptive beamforming - mainly due to the large errors occurring in the received DWTC. As expected, significant performance gains in the transmission of compressed images is achieved with an OFDM system using space-time coding and adaptive beamforming.

\section{CONCLUSION}

In this paper, we have introduced a transmission system for the application of compressed images over wireless channels using a combined source coding and STBC-OFDMAB scheme. Simulation results based on the application of digital still images, show a good match between objective performance metrics and subjective fidelity measures (human 


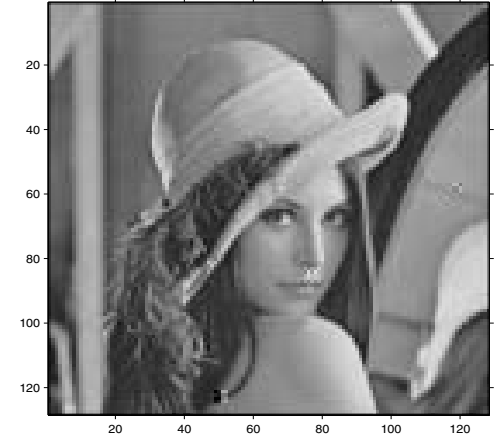

Fig. 5. Reconstructed Lena image from the OFDM system with no spacetime coding and no adaptive beamforming: $S N R=20 \mathrm{~dB}$

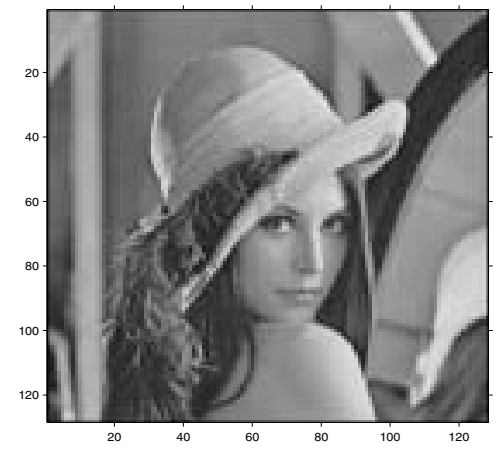

Fig. 6. Reconstructed Lena image from the OFDM system with space-time coding and adaptive beamforming: $\mathrm{SNR}=10 \mathrm{~dB}$

visual perception). Finally, this initial study confirms that wireless comunication systems based on adaptive space-time OFDM are better suited to multimedia communication.

Currently, we are working on an extension of this research study, which focuses on the optimization of various compression parameters using an objective criterion such as the system SNR. Determining an optimal choice of a wavelet basis function, which aims to improve overall compression performance, has also become essential in improving the channel capacity of ST-OFDM systems to support high data rate multimedia applications.

\section{REFERENCES}

[1] J. F. Huber, "Mobile next-generation networks", IEEE Multimedia, vol. 11, pp. 72-83, Jan.-Feb. 2004.

[2] T. S. Rappaport, "Wireless Communications Principles and Practice," 2nd ed., Delhi, India: Pearson Education Inc., 2002.

[3] M. Ghanbari, "Standard Codecs: Image Compression to Advanced Video Coding," Herts, UK: The Institution of Electrical Engineers, IEE Telecommunications Series 49, 2003.

[4] C. N. Taylor and S. Dey, "Adaptive image compression for wireless multimedia communication," in IEEE ICC, 2001 pp. 1925 - 1929.

[5] J. Yang, M. H. Lee, M. Jiang, and J. Y. Park, "Robust wireless image transmission based on turbo-coded OFDM," IEEE Transactions on Consumer Electronics, vol. 48, pp. 724-730, Aug. 2002.

[6] S. M. Alamouti, "A simple transmit diversity technique for wireless communications," IEEE J. Select. Areas Commun., vol. 16, pp. 14511458, Oct. 1998.

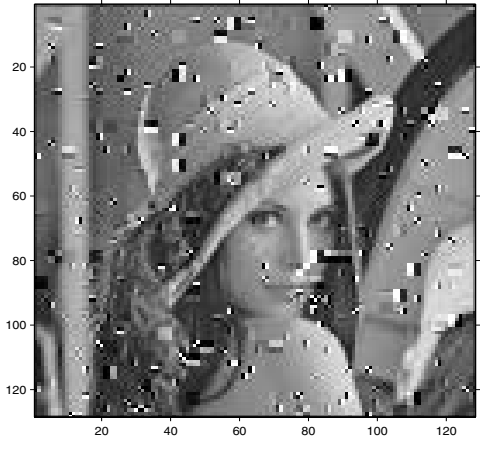

Fig. 7. Reconstructed Lena image from the OFDM system with no spacetime coding and no adaptive beamforming: $S N R=10 \mathrm{~dB}$

[7] V. Tarokh, H. Jafarkhani, and A. R. Calderbank, "Space-time block codes from orthogonal designs," IEEE Trans. Inform. Theory, vol. 45, pp. 1456-1467, Jul. 1998.

[8] K. H. Lin, Z. M. Hussain, and R. Harris, "Adaptive transmit eigenbeamforming with orthogonal space-time block coding in correlated spacetime channels," in Proc. IEEE ICASSP, 2004, pp. 817-820.

[9] K. H. Lin, Z. M. Hussain, and R. J. Harris, "Space-time OFDM with adaptive beamforming: performance in spatially correlated channels," in Proc. IEEE TENCON, 2004, pp. 617-620.

[10] T. Athanasiadis, K. H. Lin, and Z. M. Hussain, "Space-time OFDM with Adaptive Beamforming for Wireless Multimedia Applications," Proc. IEEE International Conference on Information Technology and Applications (ICITA 2005), Sydney, Jul. 2004.

[11] M. Kr. Mandal, "Multimedia Signals and Systems," Massachusetts, USA: Kluwer Academic, 2003.

[12] J. -R. Ohm, "Multimedia Communication Technology: Representation, Transmission and Identification of Multimedia Signals," Germany: Springer-Verlag Berlin Heidelberg, 2004.

[13] Z. Xiang, K. Ramchandran, M. T. Orchard, and Y. Q. Zhang, ”A comparative study of DCT and wavelet-based image coding," IEEE Trans. Circuits Syst. Video Technol, vol. 9, pp. 692-695, Apr. 1999.

[14] K. Skretting, J. H. Husoy, and S. O. Aase, "Improved huffman coding using recursive splitting," NORSIG 1999, Proc. Norwegian Signal Proc. Symp., Asker, Norway, Sept. 1999.

[15] Siemens, Channel Model for Tx Diversity Simulations using Correlated Antennas, 3GPP Document TSG-RAN WG1 \#15, R1-00-1067, Berlin, Germany, Aug. 2000.

[16] S. S. Mahmoud, Z. M. Hussain, and P. O'Shea, "A space-time model for mobile radio channel with hyperbolically distributed scatterers," IEEE Antennas and Wireless Propagation Letters, vol. 1, pp. 211-214, 2002. 